\title{
Variability of Cometary X-Ray Emission Induced by Solar Wind lons
}

\section{Citation}

Kharchenko, V., and A. Dalgarno. 2001. "Variability of Cometary X-Ray Emission Induced by Solar Wind lons." The Astrophysical Journal 554 (1): L99-102. https://doi.org/10.1086/320929.

\section{Permanent link}

http://nrs.harvard.edu/urn-3:HUL.InstRepos:41397461

\section{Terms of Use}

This article was downloaded from Harvard University's DASH repository, and is made available under the terms and conditions applicable to Other Posted Material, as set forth at http:// nrs.harvard.edu/urn-3:HUL.InstRepos:dash.current.terms-of-use\#LAA

\section{Share Your Story}

The Harvard community has made this article openly available.

Please share how this access benefits you. Submit a story.

\section{Accessibility}


The Astrophysical Journal, 554:L99-L102, 2001 June 10

(C) 2001. The American Astronomical Society. All rights reserved. Printed in U.S.A.

\title{
VARIABILITY OF COMETARY X-RAY EMISSION INDUCED BY SOLAR WIND IONS
}

\author{
V. Kharchenko and A. Dalgarno \\ Harvard-Smithsonian Center for Astrophysics, 60 Garden Street, Cambridge, MA 02138; \\ vkharchenko@cfa.harvard.edu, adalgarno@cfa.harvard.edu \\ Received 2001 March 23; accepted 2001 May 4; published 2001 June 6
}

\begin{abstract}
X-ray emission spectra induced by the interaction of the slow and fast solar wind components with cometary gas are calculated for typical solar wind compositions. The emission spectra arising from the charge transfer mechanism are shown to be in good agreement with observational data. The identities and intensities of the brightest spectral lines that include forbidden transitions are obtained for the slow and fast solar winds. Differences in emission spectra of individual comets occur because of variations in the solar wind composition. Comparisons with observational data for comets Levy and Hale-Bopp indicate that they were subjected to the slow solar wind. The spectra at photon energies above $500 \mathrm{eV}$ fluctuate with the solar wind composition because of the varying presence of fully stripped oxygen ions. Mechanisms of X-ray photon emission at energies above $0.9 \mathrm{keV}$ are discussed.
\end{abstract}

Subject headings: atomic processes — comets: general — X-rays: general

\section{INTRODUCTION}

X-ray and extreme-ultraviolet (EUV) radiation has been observed from more than a dozen comets (Lisse et al. 1996, 1999a, 1999b; Dennerl, Englhauser, \& Trümper 1997; Mumma, Krasnopolsky, \& Abbott 1997; Krasnopolsky et al. 1997; Owens et al. 1998; Krasnopolsky, Mumma, \& Abbott 2000; Krasnopolsky \& Mumma 2001). Of the several possible excitation mechanisms (Wickramasinghe \& Hoyle 1996; Cravens 1997; Bingham et al. 1997, 2000; Northrop et al. 1997; Ip \& Chow 1997; Uchida et al. 1998; Shapiro et al. 1999), the charge transfer of solar wind ions in collisions with the cometary atmosphere (Cravens 1997) appears to be the most plausible (Häberli et al. 1997; Mumma et al. 1997; Krasnopolsky 1997; Wegmann et al. 1998; Kharchenko \& Dalgarno 2000; Schwadron \& Cravens 2000). If the charge transfer mechanism dominates the excitation, then with sufficient spectral resolution the composition of the solar wind can be inferred from a comparison of the observed spectra with the spectra predicted from charge transfer (Dennerl et al. 1997; Kharchenko \& Dalgarno 2000; Schwadron \& Cravens 2000).

A model spectrum based on the assumptions that the solar wind ions behave as hydrogenic systems and only allowed radiative transitions occur has been constructed by Schwadron $\&$ Cravens (2000). They incorporate the contributions from the impacts of the fast solar wind and the slow solar wind that have different ion compositions. From a comparison with the observational data (Dennerl et al. 1997), Schwadron \& Cravens (2000) concluded that comet C/1990 K1 (Levy) was subjected primarily to the fast solar wind. We employ a more detailed description of the capture and radiative processes and argue that X-ray emissions from comets C/1990 K1 (Levy), P/Encke 1997, and C/1995 O1 (Hale-Bopp) were induced by slow wind ions. We demonstrate that forbidden transitions give rise to intense emission lines, which, because of the long lifetimes of metastable states, are absent from laboratory beam spectra (Suraud et al. 1991; Greenwood et al. 2000).

\section{RADIATIVE TRANSITIONS}

Highly charged ions moving at solar wind velocities capture electrons into a range of excited states with a distribution that depends on the charge and energy of the ion and on the identity of the target species that, for comets, is primarily the water molecule with lesser abundances of $\mathrm{CO}, \mathrm{CO}_{2}, \mathrm{OH}, \mathrm{O}$, and $\mathrm{H}$. Multielectron charge transfer can occur at low collision velocities (Posthumus \& Morgenstern 1992; Kamber et al. 1999; Beiersdorfer et al. 2000; Greenwood et al. 2000; Moretto-Capelle, Bordenave-Montesquieu, \& Bordenave-Montesquieu 2000), but the resulting excited ions decay mostly by emission of energetic Auger electrons (Posthumus \& Morgenstern 1992; Kamber et al. 1999; Moretto-Capelle et al. 2000) and rarely produce Xrays. X-ray emission arises from single-electron capture into specific excited states (Janev \& Winter 1985; Suraud et al. 1991; Janev 1995; Greenwood et al. 2000; Beiersdorfer et al. 2000). To calculate the populations of the excited states, we used energydependent cross sections for the population of individual states taken from the literature (Kharchenko \& Dalgarno 2000). Electron capture occurs preferentially into highly excited states, and states of lower energy are then populated by radiative cascading transitions. The cascading pathways are controlled by the branching ratios of individual transition probabilities, and the number of cascading photons is large (Kharchenko, Liu, \& Dalgarno 1998; Kharchenko \& Dalgarno 2000).

Cascading photons contain important information on solar wind ion composition. Thus, theoretical models predicted very different relative intensities of cascading photons for hydrogenlike and helium-like ions because of the spin selection rules for radiative transitions (Kharchenko et al. 1998; Kharchenko \& Dalgarno 2000). Recent laboratory measurements of X-ray spectra induced in charge transfer collisions demonstrate these differences (Beiersdorfer et al. 2000). Of particular interest are the helium-like ions formed by capture of electrons by hydrogenlike ions. Approximately three-quarters of the captures occur into triplet states that, for the $\mathrm{O}^{+6}, \mathrm{~N}^{+5}$, and $\mathrm{C}^{+4}$ ions, radiate preferentially by allowed cascading transitions into metastable $2^{3} \mathrm{~S}$ states or excited triplet $2^{3} \mathrm{P}$ states. The $2^{3} S$ states decay into the ground $1^{1} S$ state by relativistic magnetic dipole transitions (Drake 1971). For $\mathrm{C}^{+4}$, the $2^{3} S \rightarrow 1^{1} S$ transition has an energy of 298.89 $\mathrm{eV}$ and a decay time of $0.02 \mathrm{~s}$, and for helium-like $\mathrm{N}^{+5}$, an energy of $419.79 \mathrm{eV}$ and a decay time of $3.9 \times 10^{-3} \mathrm{~s}$. X-ray emissions from long-lived metastable states are difficult to detect in laboratory ion-beam experiments (Greenwood et al. 2000) 

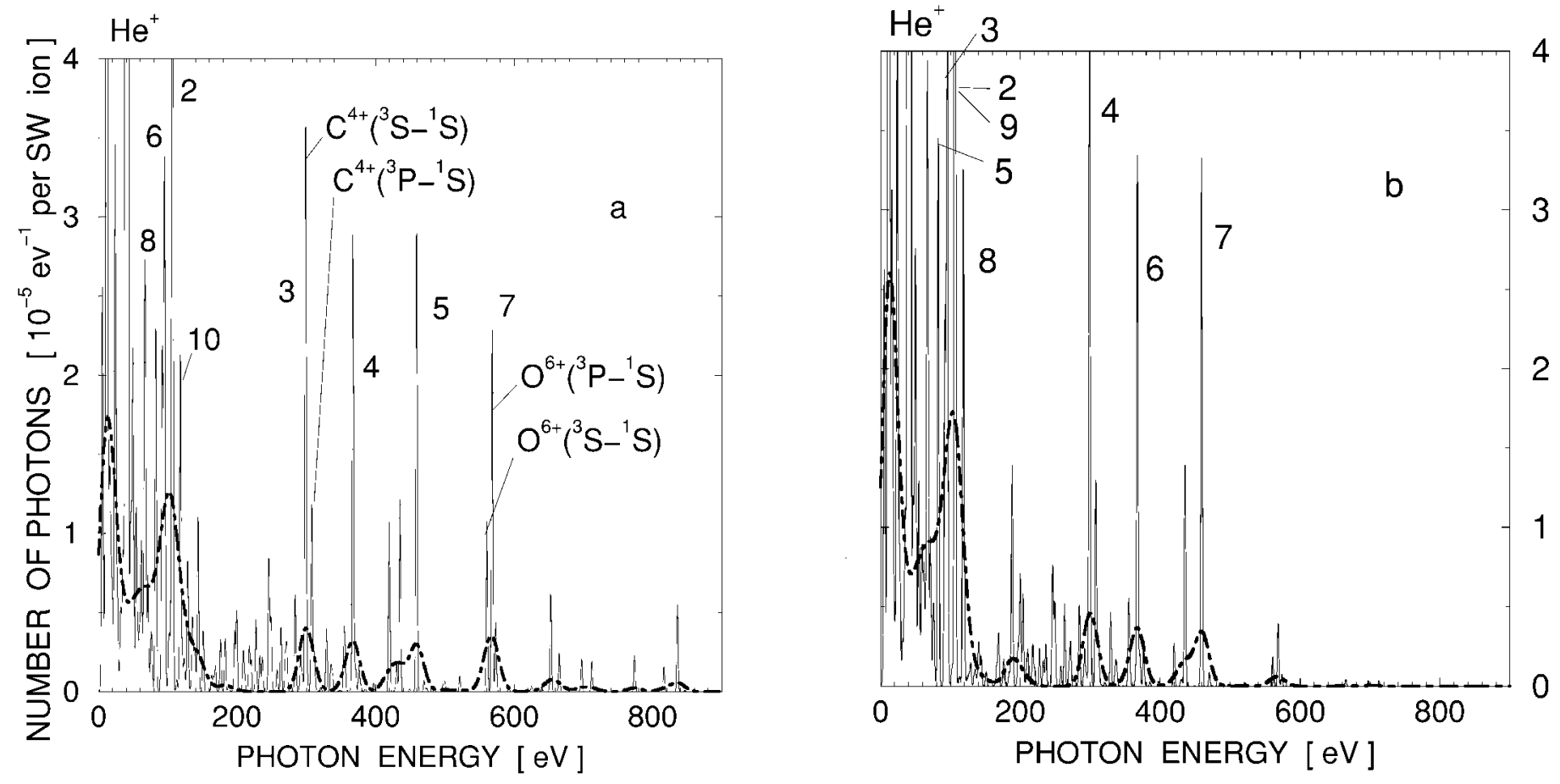

FIG. 1.-Cometary emission spectra induced in charge transfer collisions of heavy ions of the slow (left) and fast (right) solar wind (SW). The emission spectra with energy resolution of $1 \mathrm{eV}$ are indicated by solid lines. The dot-dashed lines show the emission spectra from lines of $\mathrm{O}, \mathrm{C}, \mathrm{N}$, and Ne ions only at a photon energy resolution of $10 \mathrm{eV}$. The emission lines from the ${ }^{3} S$ and ${ }^{3} P$ states of $\mathrm{C}^{+4}$ and of $\mathrm{O}^{+6}$ are indicated in the left panel. The numbers refer to the transitions listed in Tables 1 and 2 .

because, at typical velocities of $500 \mathrm{~km} \mathrm{~s}^{-1}$, the distances required for metastable $2^{3} S$ ions to decay to the $1^{1} S$ state, $10 \mathrm{~km}$ for $\mathrm{C}^{+5}$, $2 \mathrm{~km}$ for $\mathrm{N}^{+5}$, and $0.5 \mathrm{~km}$ for $\mathrm{O}^{+6}$, are much larger than the detector dimensions.

For ions heavier than $\mathrm{C}^{+4}$, the $2^{3} P_{1}$ state decays more frequently by spin-forbidden transitions directly to the ground state, reducing the intensity of the $2^{3} S \rightarrow 1^{1} S$ transitions (Drake \& Dalgarno 1969; Lin, Johnson, \& Dalgarno 1977). The $2^{3} P_{1} \rightarrow 1^{1} S$ transition of $\mathrm{O}^{+6}$ lying at $568.4 \mathrm{eV}$ is more intense than the $2^{3} S \rightarrow 1^{1} S$ line at $560.9 \mathrm{eV}$. The radiative lifetimes are shorter than the collisional lifetimes in dilute cometary atmospheres so that, with the exception of the $2^{3} S$ of neutral helium populated by the sequential charge transfer of $\mathrm{He}^{+2}$ ions, collisional redistribution does not occur. The $2^{3} \mathrm{~S}$ state of $\mathrm{He}$ has a radiative lifetime of $2.2 \mathrm{hr}$ (Drake 1971) and will be quenched by collisions.

TABLE 1

Most Intense Lines Induced by the Slow Solar Wind

\begin{tabular}{lcccc}
\hline \hline Number & Ion & Transition & $\begin{array}{c}\text { Photon Energy } \\
(\mathrm{eV})\end{array}$ & Intensity $^{\mathrm{a}}$ \\
\hline $1 \ldots \ldots$. & $\mathrm{O}^{+5}$ & $1 s^{2} 2 p^{2} P \rightarrow 1 s^{2} 2 s^{2} S$ & 11.99 & $2.49 \times 10^{-4}$ \\
$2 \ldots \ldots$. & $\mathrm{O}^{+5}$ & $1 s^{2} 5 d^{2} D \rightarrow 1 s^{2} 2 p{ }^{2} P$ & 106.5 & $1.21 \times 10^{-4}$ \\
$3 \ldots \ldots$. & $\mathrm{C}^{+4}$ & $1 s 2 s^{3} S \rightarrow 1 s^{2}{ }^{1} S$ & 298.89 & $8.88 \times 10^{-5}$ \\
$4 \ldots \ldots$. & $\mathrm{O}^{+5}$ & $2 p{ }^{2} P \rightarrow 1 s^{2} S$ & 367.3 & $7.31 \times 10^{-5}$ \\
$5 \ldots \ldots$. & $\mathrm{O}^{+5}$ & $4 p^{2} P \rightarrow 1 s^{2} S$ & 459.16 & $7.26 \times 10^{-5}$ \\
$6 \ldots \ldots$. & $\mathrm{O}^{+5}$ & $1 s^{2} 4 d^{2} D \rightarrow 1 s^{2} 2 p^{2} P$ & 95.48 & $6.83 \times 10^{-5}$ \\
$7 \ldots \ldots$. & $\mathrm{O}^{+6}$ & $1 s 2 p^{3} P \rightarrow 1 s^{2}{ }^{2} S$ & 568.4 & $5.75 \times 10^{-5}$ \\
$8 \ldots \ldots$. & $\mathrm{O}^{+5}$ & $1 s^{2} 3 p^{2} P \rightarrow 1 s^{2} 2 s^{2} S$ & 82.59 & $5.63 \times 10^{-5}$ \\
$9 \ldots \ldots$. & $\mathrm{C}^{+4}$ & $1 s 2 p{ }^{3} P \rightarrow 1 s 2 s^{3} S$ & 5.44 & $5.54 \times 10^{-5}$ \\
$10 \ldots \ldots$ & $\mathrm{O}^{+5}$ & $1 s^{2} 5 p^{2} P \rightarrow 1 s^{2} 2 s^{2} S$ & 118.28 & $5.31 \times 10^{-5}$ \\
\hline
\end{tabular}

${ }^{a}$ Line intensity corresponds to unit solar wind flux.

\section{EMISSION SPECTRA}

Oxygen and carbon ions together with neon and nitrogen ions are responsible for the emission of most of the energetic photons. Heavier ions, iron, sulfur, silicon, and magnesium, are less abundant and tend to produce photons at soft X-ray and EUV wavelengths (Schwadron \& Cravens 2000). In our calculations, the emissions of heavier ions were approximated by hydrogenic spectra with accurate branching ratios for the cascading photons (Kharchenko et al. 1998; Kharchenko \& Dalgarno 2000). They differ significantly from spectra obtained with the equal probability model (Wegmann et al. 1998; Schwadron \& Cravens 2000).

We have calculated the spectra corresponding to two different compositions of the solar wind. The fractional abundances of the positively charged ions $\mathrm{O}, \mathrm{C}, \mathrm{N}, \mathrm{Ne}, \mathrm{Fe}, \mathrm{S}, \mathrm{Si}$, and $\mathrm{Mg}$ characteristic of the fast and slow solar wind components (von Steiger et al. 1992, 2000; Schwadron \& Cravens 2000) are listed by Schwadron \& Cravens (2000). The resulting line spectra are shown in Figure 1, and the relative strengths of the brightest emission lines normalized to the unit solar wind flux are given in Tables 1 and 2. The strongest lines in the emission spectra are induced by oxygen and carbon ions, which are the most abundant heavy ion constituents in both the slow wind and the fast wind. Some of the brightest lines arise in the spin-forbidden transitions of helium-like ions of $\mathrm{O}^{+6}$ and $\mathrm{C}^{+4}$. The $2^{3} \mathrm{P} \rightarrow 1^{1} S$ line of $\mathrm{O}^{+6}$ at $568.4 \mathrm{eV}$ is 2.5 times more intense than the $2^{3} S \rightarrow 1^{1} S$ line at $560.9 \mathrm{eV}$. Lines from the same spin-forbidden transitions in $\mathrm{C}^{+4}$ have the opposite intensity ratios: the $2^{3} P \rightarrow 1^{1} S$ line at $304.6 \mathrm{eV}$ is about 3.5 times less intense than the $2^{3} S \rightarrow 1^{1} S$ line at $298.9 \mathrm{eV}$. These features 
TABLE 2

Most Intense Lines Induced by the Fast Solar Wind

\begin{tabular}{lcccc}
\hline \hline Number & Ion & Transition & $\begin{array}{c}\text { Photon Energy } \\
(\mathrm{eV})\end{array}$ & Intensity \\
\hline $1 \ldots \ldots$. & $\mathrm{O}^{+5}$ & $1 s^{2} 2 p^{2} P \rightarrow 1 s^{2} 2 s^{2} S$ & 11.99 & $3.81 \times 10^{-4}$ \\
$2 \ldots \ldots \ldots$ & $\mathrm{O}^{+5}$ & $1 s^{2} 5 d^{2} D \rightarrow 1 s^{2} 2 p^{2} P$ & 106.5 & $1.85 \times 10^{-4}$ \\
$3 \ldots \ldots$. & $\mathrm{O}^{+5}$ & $1 s^{2} 4 d^{2} D \rightarrow 1 s^{2} 2 p^{2} P$ & 95.48 & $1.04 \times 10^{-4}$ \\
$4 \ldots \ldots$. & $\mathrm{C}^{+4}$ & $1 s 2 s^{3} S \rightarrow 1 s^{2}{ }^{2} S$ & 298.89 & $1.01 \times 10^{-4}$ \\
$5 \ldots \ldots$. & $\mathrm{O}^{+5}$ & $1 s^{2} 3 p^{2} P \rightarrow 1 s^{2} 2 s^{2} S$ & 82.59 & $8.59 \times 10^{-5}$ \\
$6 \ldots \ldots$. & $\mathrm{O}^{+5}$ & $2 p^{2} P \rightarrow 1 s^{2} S$ & 367.3 & $8.38 \times 10^{-5}$ \\
$7 \ldots \ldots$. & $\mathrm{O}^{+5}$ & $4 p^{2} P \rightarrow 1 s^{2} S$ & 459.16 & $8.33 \times 10^{-5}$ \\
$8 \ldots \ldots$. & $\mathrm{O}^{+5}$ & $1 s^{2} 5 p^{2} P \rightarrow 1 s^{2} 2 s^{2} S$ & 118.28 & $8.11 \times 10^{-5}$ \\
$9 \ldots \ldots$. & $\mathrm{O}^{+5}$ & $1 s^{2} 4 p^{2} P \rightarrow 1 s^{2} 2 s^{2} S$ & 107.04 & $7.83 \times 10^{-5}$ \\
$10 \ldots \ldots$ & $\mathrm{C}^{+4}$ & $1 s 2 p^{3} P \rightarrow 1 s 2 s^{3} S$ & 5.44 & $6.36 \times 10^{-5}$ \\
\hline
\end{tabular}

provide a unique diagnostic probe of the source of X-ray emission in cometary atmospheres.

There are significant differences in the emission spectra of the slow wind (Fig. 1, left) and the fast wind (Fig. 1, right) with an absence of energetic photons for the fast wind. The slow wind contains larger fractions of highly charged $\mathrm{O}^{+8}, \mathrm{O}^{+7}$, and $\mathrm{C}^{+6}$ ions that yield $\mathrm{X}$-ray photons with energies above $450 \mathrm{eV}$. The abundance of bare oxygen ions in the solar wind is particularly changeable, and we anticipate large variations in the intensity of energetic X-ray photons above $600 \mathrm{eV}$.

The ions of the heavier elements $\mathrm{Fe}, \mathrm{S}, \mathrm{Si}$, and $\mathrm{Mg}$ contribute importantly to X-ray cometary spectra in the photon energy range of 200-300 eV where there are few lines from oxygen and carbon ions. This is apparent from the spectrum with an energy resolution of $10 \mathrm{eV}$ shown in Figure 1 for $\mathrm{O}, \mathrm{C}, \mathrm{N}$, and $\mathrm{Ne}$ ions only. The resonant emission line of excited $\mathrm{He}^{+}$produced in electron-capture collisions of $\mathrm{He}^{+2}$ solar wind ions is also indicated in Figure 1. The $\mathrm{He}^{+}$line will be one of the brightest lines in the ultraviolet emission spectrum, because the fraction of $\mathrm{He}^{+2}$ ions is about 100 times larger than oxygen and carbon ions, and it accounts for $4 \%-5 \%$ of the total ion flux (von Steiger et al. 1992, 2000). At low spectral resolution, the very intense line of $\mathrm{He}^{+}$at $40.82 \mathrm{eV}$ may significantly modify the envelope of the EUV and soft X-ray photons.

To demonstrate the influence of the energy resolution on the shape of spectral curves, we have calculated emission spectra at resolutions of 1, 10, and $50 \mathrm{eV}$ for the slow solar wind. The spectra are shown in the left panel of Figure 2. Maxima occur in the spectral envelopes that are formed by groups of unresolved lines and not by individual spectral lines.

In the right panel of Figure 2, the predicted emission spectra are compared with observations of X-ray and UV emissions from the comets P/Encke 1997 and C/1995 O1 (Hale-Bopp) (Owens et al. 1998; Lisse et al. 1999a). The observed spectra of X-rays at an energy resolution of $50 \mathrm{eV}$ of the two comets are in disagreement with the spectrum calculated for the fast wind at energies above $500 \mathrm{eV}$ but agree satisfactorily at all energies with the spectrum for the slow wind. The right panel of Figure 2 includes the low-resolution fitted spectra obtained from comet C/1990 K1 (Levy) as given by Dennerl et al. (1997). Contrary to the conclusion of Schwadron \& Cravens (2000) that the spectrum of comet Levy supports the fast solar wind origin, we find it to be consistent with the slow wind. The slow wind compositions that we infer are expected from the low-latitude heliospheric positions of the comets during the periods of the observations (Lisse et al. 1999a; von Steiger et al. 2000; Schwadron \& Cravens 2000).

The profiles of the low-energy part of observed and predicted spectra are largely due to the contribution of the $\mathrm{He}^{+}$resonance line, indicated by the solid curves in the right panel of Figure 2. At an energy resolution of $50 \mathrm{eV}$, the spectra of the fast and slow wind emissions in the right panel of Figure 2 are similar at photon energies less than $400 \mathrm{eV}$. Above $400 \mathrm{eV}$, the fast wind spectra show many fewer energetic X-ray photons and are easily distinguished from the slow wind emission.

We suggested earlier that an energy resolution of $10 \mathrm{eV}$ is adequate to distinguish between bremsstrahlung and the charge transfer mechanisms (Kharchenko \& Dalgarno 2000). Maxima
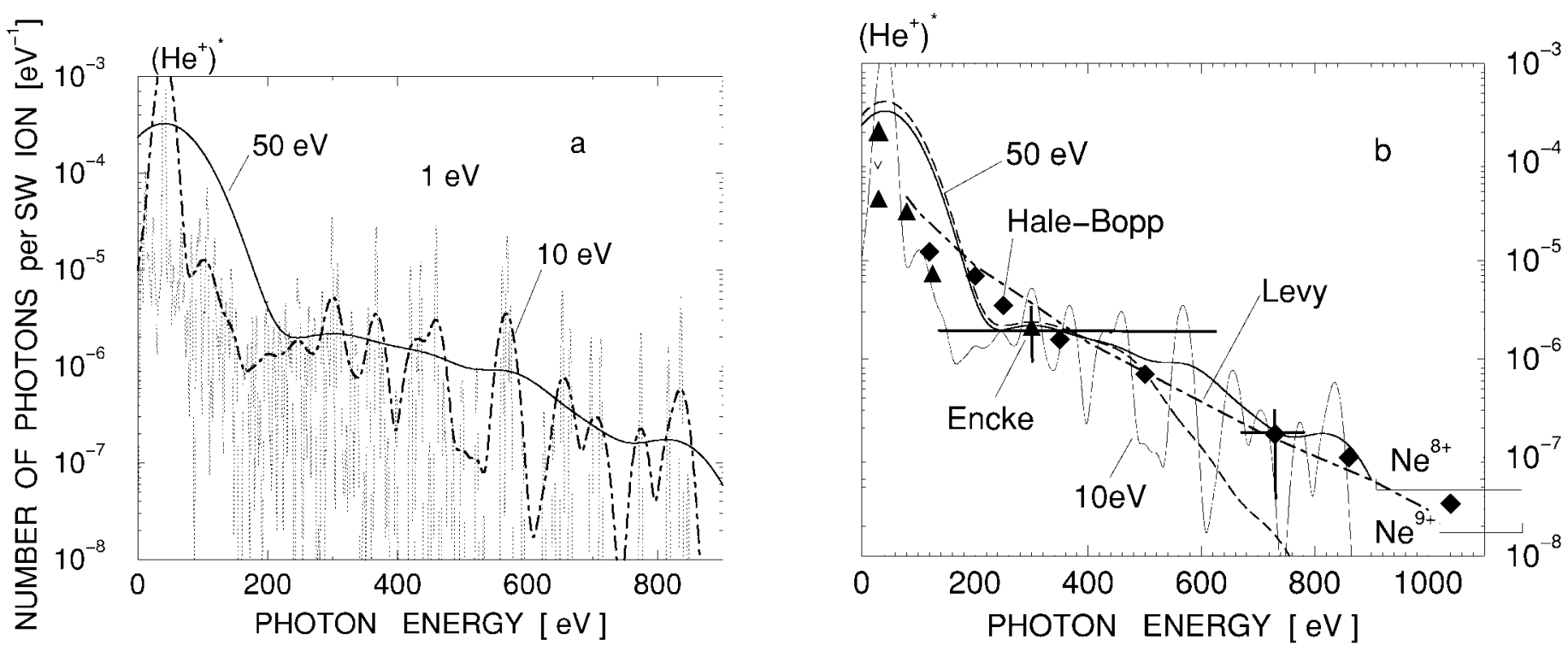

FIG. 2.-Left: Ion emission spectra induced by the slow solar wind (SW) shown for photon energy resolutions of 1 , 10 , and 50 eV as the dotted, dot-dashed,

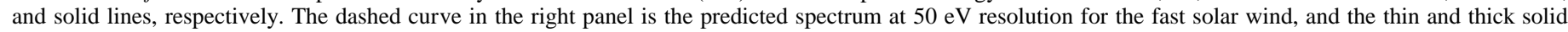

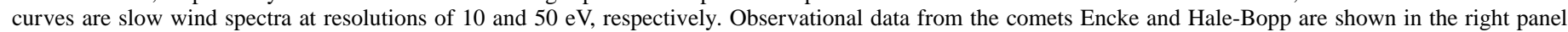

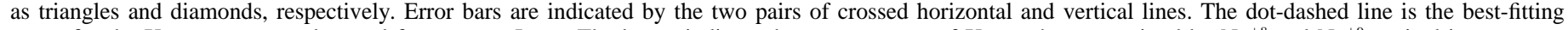
curve for the X-ray spectrum observed from comet Levy. The boxes indicate the energy range of X-ray photons emitted by $\mathrm{Ne}^{+8}$ and $\mathrm{Ne}^{+9}$ excited ions. 
in the spectral curves from charge transfer are formed by groups of lines as shown in Figure 2. Measurements of the relative intensities and positions of the maxima would be additional tests of the charge transfer mechanism and would provide the composition of the solar wind.

X-rays with energies larger than $0.9 \mathrm{keV}$ have been obtained in observations of several comets (Lisse et al. 1996, 1999a; Dennerl et al. 1997; Owens et al. 1998). Photons at energies between 0.91 and $1.16 \mathrm{keV}$ are emitted by excited $\mathrm{Ne}^{+8}$ ions and between 1.02 and $1.35 \mathrm{keV}$ by excited $\mathrm{Ne}^{+9}$ ions. The excited ions can be produced by electron capture by the fraction of $\mathrm{Ne}^{+9}$ and $\mathrm{Ne}^{+10}$ ions that may be present in the solar wind. High-energy X-rays can also be created following the production of K-shell vacancies in the projectile and target systems (Frémont et al. 1996), and still more energetic photons may result from colliding $\mathrm{Fe}, \mathrm{Si}, \mathrm{S}$, and $\mathrm{Mg}$ ions.

\section{CONCLUSIONS}

The low-resolution spectra and the total intensities of X-ray and EUV emissions detected from comets are reproduced by calculations with typical ion compositions of the solar wind. Differences in X-ray emission spectra of individual comets may be attributed to variations in the composition and charges of heavy ions in the solar wind.

The cometary X-ray spectra at photon energies above $450 \mathrm{eV}$ are strongly influenced by variations in the fraction of fully stripped oxygen ions in the wind. Photons with energies above $0.9 \mathrm{keV}$ may be induced by neon and heavier solar wind ions through inner-shell vacancies produced in collisions with cometary gas. Hydrogen-like models yield a general description of low-resolution spectra (Schwadron \& Cravens 2000) but do not reproduce specific features such as the widths, positions, and relative intensities of features that appear at resolutions better than $20 \mathrm{eV}$.

Populated efficiently via cascading, the metastable states of excited $\mathrm{O}^{+6}$ and $\mathrm{C}^{+4}$ ions produce bright lines. Some of the brightest emissions arise from spin-forbidden radiative transitions, which may be not be present in ion-beam experiments. The positions and relative brightnesses of the most intense lines distinguish between the slow and fast solar wind compositions.

Our calculations described cometary X-ray emission spectra from dilute atmospheres, where photon cascading occurs between sequential collisions. Cascading without collisional interference occurs in the atmospheres of large comets with high gas production rates. For them, the brightest area of cometary emission is shifted from the cometary core by about $15,000-20,000 \mathrm{~km}$ in the sunward direction. For small cometary atmospheres, the brightest region of X-ray emission is the cometary center. In the dense cores of cometary atmospheres, ion collisions with neutral atoms and molecules may be frequent and may significantly reduce the populations of long-lived metastable states, diminishing the intensities of spectral lines arising from forbidden transitions.

This work has been supported by the National Aeronautic and Space Administration under grant NAG5-4986.

\section{REFERENCES}

Beiersdorfer, P., et al. 2000, Phys. Rev. Lett., 85, 5090

Bingham, R., Dawson, J. M., Shapiro, V. D., Mendis, D. A., \& Kellet, B. 1997, Science, 271, 49

Bingham, R., Kellet, B., Dawson, J. M., Shapiro, V. D., \& Mendis, D. A. 2000, ApJS, 127, 233

Cravens, T. E. 1997, Geophys. Res. Lett., 24, 105

Dennerl, K., Englhauser, J., \& Trümper, J. 1997, Science, 277, 1625

Drake, G. W. F. 1971, Phys. Rev. A, 3, 908

Drake, G. W. F., \& Dalgarno, A. 1969, Mem. Soc. R. Sci. Liège, 17, 69

Frémont, F., Bedouet, C., Chesnel, J. Y., Merabet, H., \& Husson, X. 1996, Phys. Rev. A, 54, R4609

Greenwood, J. B., Williams, I. D., Smith, S. J., \& Chutjian, A. 2000, ApJ, 533, L175

Häberli, R. M., Gombosi, T. I., DeZeuuw, D. L., Combi, M. R., \& Powell, K. G. 1997, Science, 276, 939

Ip, W.-H., \& Chow, V. W. 1997, Icarus, 130, 217

Janev, R. K., ed. 1995, Atomic and Molecular Processes in Fusion Edge Plasmas (New York: Plenum)

Janev, R. K., \& Winter, H. 1985, Phys. Rep., 117, 265

Kamber, E. Y., Abdallah, M. A., Cocke C. L., \& Stöckli, M. 1999, Phys. Rev. A, 60, 2907

Kharchenko, V., \& Dalgarno, A. 2000, J. Geophys. Res., 105, 18,351

Kharchenko, V., Liu, W., \& Dalgarno, A. 1998, J. Geophys. Res., 103, 26,687

Krasnopolsky, V. 1997, Icarus, 188, 368

Krasnopolsky, V. A., et al. 1997, Science, 277, 1488

Krasnopolsky, V. A., \& Mumma, M. J. 2001, ApJ, 549, 629
Krasnopolsky, V. A., Mumma, M. J., \& Abbott, M. J. 2000, Icarus, 146, 152 Lin, C. D., Johnson, W. R., \& Dalgarno, A. 1977, Phys. Rev. A, 15, 154

Lisse, C. M., et al. 1996, Science, 274, 205

1999a, Earth Moon Planets, 77, 283

1999, Icarus, 141, 316

Moretto-Capelle, P., Bordenave-Montesquieu, D., \& Bordenave-Montesquieu, A. 2000, J. Phys. B, 33, L735

Mumma, M. J., Krasnopolsky, V. A., \& Abbott, M. J. 1997, ApJ, 491, L125

Northrop, T. G., Lisse, C. M., Mumma, M. J., \& Desch, M. D. 1997, Icarus, 127,246

Owens, A., et al. 1998, ApJ, 493, L47

Posthumus, J. H., \& Morgenstern, R. 1992, Phys. Rev. Lett., 68, 1315

Schwadron, N. A., \& Cravens, T. E. 2000, ApJ, 544, 558

Shapiro, V. D., Bingham, R., Dawson, J. M., Dobe, Z., Kellett, B. J., \& Mendis, D. A. 1999, J. Geophys. Res., 104, 2537

Suraud, M. G., Hoekstra R., de Heer, F. J., Bonnet, J. J., \& Morgenstern, R. 1991, J. Phys. B, 24, 2543

Uchida, M. M., Morikawa, M., Kubotani, H., \& Mouri, H. 1998, ApJ, 498, 863

von Steiger, R., Christon, S. P., Gloeckler, G., \& Ipavich, F. M. 1992, ApJ, 389, 791

von Steiger, R., et al. 2000, J. Geophys. Res., 105, 27,217

Wegmann, R., Schmidt, H. U., Lisse, C. M., Dennerl, K., \& Englhauser, J. 1998, Planet. Space Sci., 46, 603

Wickramasinghe, N. C., \& Hoyle, F. 1996, Ap\&SS, 239, 121 